\title{
Cdk5 Regulates the Phosphorylation of Tyrosine 1472 NR2B and the Surface Expression of NMDA Receptors
}

\author{
Shuo Zhang, Lambert Edelmann, June Liu, James E. Crandall, and Maria A. Morabito \\ University of Massachusetts Medical School, Department of Cell Biology, Waltham, Massachusetts 02452
}

\begin{abstract}
NMDA receptors (NMDARs) are a major class of ionotropic glutamate receptors that can undergo activity-dependent changes in surface expression. Clathrin-mediated endocytosis is a mechanism by which the surface expression of NR2B-containing NMDA receptors is regulated. The $\mathrm{C}$ terminus of the NMDA receptor subunit NR2B contains the internalization motif YEKL, which is the binding site for the clathrin adaptor AP-2. The tyrosine (Y1472) within the YEKL motif is phosphorylated by the Src family of kinases and this phosphorylation inhibits the binding of AP-2 and promotes surface expression of NMDA receptors. Cdk 5 is a serine/threonine kinase that has been implicated in synaptic plasticity, learning, and memory. Here we demonstrate that inhibition of Cdk5 results in increased phosphorylation of Y1472 NR2B at synapses and decreased binding of NR2B to $\beta 2$-adaptin, a subunit of AP-2, thus blocking the activity-dependent endocytosis of NMDA receptors. Furthermore, we show that inhibition of Cdk5 increases the binding of Src to postsynaptic density- 95 (PSD-95), and that expression of PSD-95 facilitates the phosphorylation of Y1472 NR2B by Src. Together, these results suggest a model in which inhibition of Cdk5 increases the binding of Src to PSD-95 and the phosphorylation of Y1472 NR2B by Src, which results in decreased binding of NR2B to AP-2, and NR2B/NMDAR endocytosis. This study provides a novel molecular mechanism for the regulation of the surface expression of NR2B-containing NMDA receptors and gives insight into the Cdk5-dependent regulation of synaptic plasticity.
\end{abstract}

Key words: NMDA receptors; endocytosis; Src; PSD-95; glutamatergic synapses; Cdk5; phosphorylation

\section{Introduction}

NMDA receptors (NMDARs) are a major class of ionotropic glutamate receptors that consist of heteromeric complexes composed primarily of NR1, NR2, and NR3 subunits (Dingledine et al., 1999; Cull-Candy et al., 2001). NMDARs have been implicated in neuronal development, synaptic plasticity, learning and memory, and addiction (Dingledine et al., 1999; Kumari and Ticku, 2000), as well as psychiatric and neurodegenerative disorders (Snyder et al., 2005; Ross et al., 2006). Clathrin-mediated endocytosis is a mechanism by which the surface expression of NMDARs is regulated during synapse maturation, in long-term depression (LTD), and in response to ligand-binding (Roche et al., 2001; Snyder et al., 2001; Vissel et al., 2001; Barria and Malinow, 2002; Li et al., 2002; Montgomery and Madison, 2002; Nong et al., 2003, 2004; Prybylowski et al., 2005; Perez-Otano et al., 2006). NR2A and NR2B have distinct endocytic motifs in their $C$ terminus tails (Lavezzari et al., 2004). Whereas the $C$ terminus of the NR2B subunit of NMDARs contains a clathrin adaptor AP-2 binding site and internalization motif YEKL (Roche et al., 2001; Lavezzari et al., 2003, 2004), NR2A does not share the same YEKL

\footnotetext{
Received April 26, 2007; revised Nov. 15, 2007; accepted Nov. 29, 2007.

This work was supported by National Institutes of Health-National Institute on Drug Abuse Grant R01DA019451. We thank L.-H. Tsai, Y. Hayashi, L. Lanier, G. Schwarting, and Z. Xie for comments on this manuscript; and P. Howley, D. Ron, B. Samuels, M. Sheng, L.-H. Tsai, R. Wenthold, and Z. Xie for reagents. We also thank E. Pothos for assistance with acute brain slice preparation, L. Hassinger for assistance with confocal microscopy, and M. Mitra for technical assistance.

Correspondence should be addressed to Maria A. Morabito, University of Massachusetts Medical School, Department of Cell Biology, 200 Trapelo Road, Waltham, MA 02452. E-mail: maria.morabito@umassmed.edu. DOI:10.1523/JNEUROSCI.1900-07.2008

Copyright $\odot 2008$ Society for Neuroscience $\quad$ 0270-6474/08/280415-12\$15.00/0
}

motif and the YKKM motif in NR2A does not play an important role in endocytosis (Lavezzari et al., 2004). Tyrosine 1472 (Y1472) within the YEKL motif of NR2B is phosphorylated by the Src family of kinases (Cheung and Gurd, 2001; Prybylowski et al., 2005), and the phosphorylation of Y1472 inhibits the binding of AP-2 and promotes surface expression of NMDARs (Roche et al., 2001; Prybylowski et al., 2005).

The Src family of kinases (SFKs) has been implicated in the regulation of NMDAR-dependent synaptic transmission and plasticity and in learning and memory (Grant et al., 1992; Yu et al., 1997; Ali and Salter, 2001; Salter and Kalia, 2004). Src plays a central role in the regulation of NMDARs as indicated by the Src-dependent upregulation of NMDAR activity (Yu et al., 1997; Lu et al., 1998) and induction of NMDAR-dependent long-term potentiation (LTP) (Lu et al., 1998). Previously, it has been shown that a member of SFKs, Fyn, phosphorylates the Y1472 residue of NR2B and regulates the surface expression of NMDARs (Prybylowski et al., 2005). The phosphorylation of NMDARs by Fyn is promoted by postsynaptic density-95 (PSD95) (Tezuka et al., 1999), a member of the membrane-associated guanylate kinase family of proteins (MAGUKs) that binds directly to the NR2 subunits of NMDARs (Kornau et al., 1995; Niethammer et al., 1996; Roche et al., 2001; Lavezzari et al., 2003; Li et al., 2003). Src and other SFKs also bind PSD-95 (Tezuka et al., 1999; Chen et al., 2003; Hou et al., 2003; Kalia and Salter, 2003; Kalia et al., 2006), suggesting that the phosphorylation of NMDARs by Src and other SFKs may also be facilitated by PSD-95.

Cdk5 is a proline-directed serine/threonine kinase essential for brain development that is activated by the regulatory subunit 
p35 or p39 (Dhavan and Tsai, 2001) and is regulated by NMDAR activity (Wei et al., 2005). Cdk5 has also been implicated in drug addiction, neurodegenerative disorders, and many neuronal functions including synaptic plasticity (Bibb, 2003; Cheung et al., 2006; Hawasli et al., 2007). We have shown previously that Cdk5 phosphorylates the $\mathrm{N}$ terminus of PSD-95 in a region overlapping a Src-binding site (Morabito et al., 2004), suggesting that Cdk5, by regulating the binding of Src to PSD-95, may regulate the phosphorylation of Y1472 NR2B and the surface expression of NR2B-containing NMDARs.

\section{Materials and Methods}

Cell culture and transfections. Human embryonic kidney (HEK) 293T cells were maintained in DMEM supplemented with $10 \%$ fetal bovine serum and transfected using Lipofectamine 2000 (Invitrogen, Carlsbad, $\mathrm{CA}$ ) according to the manufacturer's instructions. cDNAs used in transfections were as follows: NR1 and NR2B (provided by Morgan Sheng, Massachusetts Institute of Technology, Cambridge, MA), Flag- NR2B and Flag-NR2B S1480A (Robert Wenthold, National Institute on Deafness and Other Communication Disorders, Bethesda, MD), Y527F Src (Peter Howley, Harvard University Medical School, Boston, MA), Y531F Fyn (Dorit Ron, University of California, San Francisco, CA), p35, Cdk5, DNCdk5 (Li-Huei Tsai, Massachusetts Institute of Technology), PSD95, AAA PSD-95 (T19A, S25A, S35A), and DDD PSD-95 (T19D, S25D, S35D). Cells were collected and lysed $18-20 \mathrm{~h}$ after transfection, at which time there was no apparent degradation of proteins or cell death.

Dissociated neuronal cultures and acute forebrain slices. Pregnant rats were killed in accordance with institutional guidelines and as approved by the University of Massachusetts Medical School Animal Care and Use Committee. Dissociated neuronal cultures were prepared from embryonic day 17 (E17) rat brains. For immunocytochemistry, dissociated hippocampal neurons were plated on poly-D-lysine and laminin at a density of $75,000 / 16 \mathrm{~mm}$ coverslip. For biochemistry, dissociated cortical neurons were plated at $6 \times 10^{6} / 10 \mathrm{~cm}$ dish, or $1 \times 10^{6} / 12$-well plate. Neurons were grown in Neurobasal medium supplemented with B27 and GlutaMax (Invitrogen) for 18-20 d in vitro (DIV). Coronal sections of adult mouse forebrain were cut $300 \mu \mathrm{m}$ thick with a vibratome (VT1000S; Leica, Nussloch, Germany) into oxygenated artificial CSF (Patel et al., 2003). Forebrain slices were transferred to dishes containing Neurobasal A medium supplemented with B27 and GlutaMax (Invitrogen) and were then incubated for $1 \mathrm{~h}$ at $32^{\circ} \mathrm{C}$ before treatment with roscovitine or DMSO.

Treatments of dissociated neuronal cultures and acute brain slices. To analyze the effect of inhibition of Cdk5, cultures were treated for $45 \mathrm{~min}$ with $5 \mu \mathrm{M}$ (neuronal cultures) or $15 \mu \mathrm{M}$ (forebrain slices) roscovitine (Calbiochem, La Jolla, CA), or DMSO as control. Treatment of forebrain slices with $5 \mu \mathrm{M}$ roscovitine for $45 \mathrm{~min}$ resulted in a reduction of Cdk5 activity ( $56.80 \pm 7.47 \%$ of control) as quantified with the phospho-Y15Cdk5 antibody (Fu et al., 2007) (data not shown). To determine whether the phosphorylation of Y1472 was dependent on SFKs, the neuronal cultures were treated with $1 \mu \mathrm{M}$ SU6656, a specific inhibitor of SFKs. The association of NR2B with the AP-2 complex was analyzed by treating dissociated neuronal cultures with $5 \mu \mathrm{M}$ roscovitine (or DMSO as control) for $45 \mathrm{~min}$, followed by priming NMDARs with $100 \mu \mathrm{M}$ glycine for $5 \mathrm{~min}$ according to Nong et al. (2003), in the presence of $5 \mu \mathrm{M}$ roscovitine, or DMSO. To analyze the effect of Cdk5 on the surface expression of the NMDARs, the cultures were pretreated with $5 \mu \mathrm{M}$ roscovitine (or DMSO as control) for $45 \mathrm{~min}$, primed with $100 \mu \mathrm{M}$ glycine ( $5 \mu \mathrm{M}$ roscovitine or DMSO) for $5 \mathrm{~min}$, and stimulated with $50 \mu \mathrm{M}$ NMDA, $100 \mu \mathrm{M}$ glycine for additional $5 \mathrm{~min}$, according to Nong et al. (2003), in the presence of $5 \mu \mathrm{M}$ roscovitine or DMSO.

Isolation of synaptosomal membrane fractions. Roscovitine-treated and control acute forebrain slices were subjected to biochemical fractionation based on the protocol of Dunah and Standaert (2001). All buffers were supplemented with protease and phosphatase inhibitors $(1 \mathrm{~mm}$ PMSF, $1 \mu \mathrm{g} / \mathrm{ml}$ aprotinin, $10 \mu \mathrm{g} / \mathrm{ml}$ leupeptin, $1 \mu \mathrm{g} / \mathrm{ml}$ pepstatin, $1 \mathrm{~mm}$ $\mathrm{Na}_{3} \mathrm{VO}_{4}$, and $50 \mathrm{~mm} \mathrm{NaF}$ ). Slices were homogenized with 12 strokes in ice-cold homogenization buffer (320 mm sucrose, 1 mM HEPES, pH7.4, 1 $\mathrm{mm} \mathrm{NaHCO}$, and $1 \mathrm{~mm}$ EDTA). Homogenates were then subjected to centrifugations to yield crude synaptosomal fractions (P2). For hypoosmotic lysis, P2 fractions were resuspended in homogenization buffer, diluted with 9 vol of ice-cold water, lysed with three strokes, and buffered by adding HEPES, pH 7.4 to $20 \mathrm{~mm}$ final concentration. The lysed P2 fractions were then centrifuged $33,000 \times g(15 \mathrm{~min})$ and the pellet (synaptosomal membrane fraction, LP1) was lysed in $1 \%$ deoxycholate (DOC) lysis buffer (150 mm NaCl, 1\% DOC, 50 mm Tris, $\mathrm{pH} 8.8$ ) for $2 \mathrm{~h}$ at $4^{\circ} \mathrm{C}$ followed by the addition of an equal volume of modified RIPA buffer [150 mm NaCl, 1 mм EDTA, 1\% (w/v) Triton X-100, 0.1\% (w/v) SDS, $50 \mathrm{~mm}$ Tris-HCl, pH 7.4] and $1 \mathrm{~h}$ incubation at $4^{\circ} \mathrm{C}$.

Lysates of cultured cells and embryonic brains. Protease and phosphatase inhibitors were freshly added to all buffers ( $1 \mathrm{~mm}$ PMSF, $1 \mu \mathrm{g} / \mathrm{ml}$ aprotinin, $10 \mu \mathrm{g} / \mathrm{ml}$ leupeptin, $1 \mu \mathrm{g} / \mathrm{ml}$ pepstatin, $1 \mathrm{~mm} \mathrm{Na}_{3} \mathrm{VO}_{4}$, and $50 \mathrm{~mm}$ $\mathrm{NaF}$ ). Cell lysates were obtained using weakly denaturing conditions as described previously (Kalia et al., 2003). Briefly, the cultured cells were lysed in $1 \%$ DOC lysis buffer ( $150 \mathrm{~mm} \mathrm{NaCl}, 1 \%$ DOC, $50 \mathrm{~mm}$ Tris, $\mathrm{pH}$ 8.8 ) for $2 \mathrm{~h}$ at $4^{\circ} \mathrm{C}$ followed by the addition of an equal volume of modified RIPA buffer [150 mm NaCl, 1 mm EDTA, 1\% (w/v) Triton X-100, $0.1 \%(\mathrm{w} / \mathrm{v})$ SDS, $50 \mathrm{~mm}$ Tris- $\mathrm{HCl}, \mathrm{pH} 7.4]$ and $1 \mathrm{~h}$ incubation at $4^{\circ} \mathrm{C}$. Genotyped brains from E17.5 Cdk5-/- and control (+/-) littermate mice were gifts from Zhigang Xie (L.-H. Tsai laboratory, Massachusetts Institute of Technology). Brain tissue was homogenized in 1\% DOC lysis buffer and treated as described above. Protein levels were determined using a Detergent Compatible Protein Assay (Bio-Rad, Hercules, CA).

Immunoprecipitations and Western blot analysis. Solubilized proteins were incubated overnight with anti-Src (Calbiochem), anti-Fyn (Millipore, Lake Placid, NY), anti-NR2B (Millipore), or nonspecific mouse (or rabbit) IgG (Calbiochem) antibodies as control. Immune complexes were isolated by protein-G Sepharose (or Protein-A) (GE Healthcare Bio-Sciences, Piscataway, NJ) for $1 \mathrm{~h}$ at $4^{\circ} \mathrm{C}$. Immunoprecipitates were separated by SDS-PAGE, transferred to polyvinylidene difluoride membranes (Millipore), and incubated with primary antibodies followed by HRP-conjugated secondary antibodies (GE Healthcare Bio-Sciences.). The following antibodies and concentrations were used: phospho-Y1472 NR2B (1:500, Millipore; 1:1000, Zymed, San Francisco, CA); NR2B (1: 1000, 1:400, Millipore; 1:500, BD PharMingen); NR1 (1:1000, Millipore); phospho-Y416 SFK (1:400; Cell Signaling, Beverly, MA); Src (1:250; Calbiochem); $\beta 2$-adaptin (1:500; BD PharMingen); PSD-95 (1:10000; Antibodies Incorporated, Davis, CA); p35 and Cdk5 (1:1000; Santa Cruz Biotechnology, Santa Cruz, CA); Actin (1:1000; Sigma, St. Louis, MO). Signals were detected with enhanced chemiluminescence (GE Healthcare Bio-Sciences) and developed on Kodak (Rochester, NY) x-ray film. The bands were digitally scanned and analyzed using NIH Image software. The ratios of coimmunoprecipitated versus immunoprecipitated proteins, phospho-Y1472 NR2B versus total NR2B, or phospho-Y416 Src versus total Src were expressed as mean \pm SEM and statistically compared using the Student's $t$ test.

Chymotrypsin assay. Treatment of cortical cultures with $5 \mu \mathrm{M}$ roscovitine (or DMSO as control) for 45 min was followed by priming with 100 $\mu \mathrm{M}$ glycine and $5 \mu \mathrm{M}$ roscovitine (or DMSO) for $5 \mathrm{~min}$ and stimulated with $50 \mu \mathrm{M}$ NMDA, $100 \mu \mathrm{M}$ glycine, and $5 \mu \mathrm{M}$ roscovitine (or DMSO) for an additional $5 \mathrm{~min}$. Live neurons were then subjected to chymotrypsin protease treatment to determine surface NR2B expression (Hall and Soderling, 1997; Fong et al., 2002). Briefly, cultured neurons were washed twice with warm HBSS buffer (Invitrogen) with 20 mм HEPES pH 7.4 and then incubated in $0.05 \mathrm{mg} / \mathrm{ml}$ chymotrypsin in the same buffer for 10 min. After three washes with warm HBSS buffer, neurons were lysed by sample buffer (6\% glycerol, 2\% SDS, 60 mu Tris, pH 6.8, 0.01\% bromophenol blue, $2 \% 2$-mercaptoethanol) and analyzed by SDS-PAGE. As a control for protein loading, the blots were probed with actin antibodies. The bands were digitally scanned and analyzed using NIH Image software. The NR2B bands were normalized against actin, plotted as mean \pm SEM, and analyzed for significance using the Student's $t$ test.

Immunocytochemistry. To visualize NMDAR surface expression, neurons were fixed after treatment (see above) with $4 \%$ paraformaldeyde, $5 \%$ sucrose in PBS with no detergent (to prevent permeabilization) for 10 min, washed with PBS, and then blocked with 10\% BSA in PBS (no detergent) for $1 \mathrm{~h}$. Surface NMDARs were stained overnight in 3\% BSA, 
PBS (no detergent) with a mouse antibody that recognizes an extracellular epitope of the NR1 subunit (NR1, 1:200; BD PharMingen). Under these nonpermeable conditions, staining of cytoplasmic proteins such as PSD-95 was greatly reduced (data not shown). After washes in PBS, neurons were permeabilized with $0.2 \%$ Triton X-100, $10 \%$ BSA in PBS and incubated overnight with a rabbit NR2B antibody (1:100; Millipore) to label all of the NR2B-containing NMDARs (i.e., those expressed on the surface and those internalized). After extensive washes in $0.1 \%$ Triton X-100 in PBS, cultures were incubated with anti-mouse (green) and anti-rabbit (red) secondary antibodies (Alexa Fluor; Invitrogen). Images were captured using Leica DM IRBE confocal microscope under identical conditions ( $40 \times$ objective, $1422 \times 1422$ pixels format, and identical settings). The level of surface expression of NR2B-containing NMDARs was determined by quantifying the number of puncta that were immunostained for NR2B (total number of receptors) compared with the number of puncta immunostained for NR2B and for NR1 (surface receptors, i.e., double labeled). The levels of NR2B-containing NMDARs surface expression, defined as the ratio of surface receptor $(\mathrm{NR} 2 \mathrm{~B}+1$ $\mathrm{NR} 1+$ ) versus total receptor (NR2B +), were calculated for all four conditions (DMSO \pm glycine/NMDA, roscovitine \pm glycine/NMDA). The degree of surface expression was expressed as mean \pm SEM and statistically compared using a two-way ANOVA with post hoc between treatment comparisons analyzed with the Tukey's honest significant difference (HSD) test (JMP version 5.1.2; SAS Institute, Cary, NC).

\section{Results}

\section{Inhibition of C $\mathrm{dk} 5$ activity increases the phosphorylation of NR2B at Y1472}

The synaptic scaffolding protein PSD-95 binds Fyn and facilitates the phosphorylation of NMDARs by Fyn (Tezuka et al., 1999). The phosphorylation of Y1472 NR2B by Fyn regulates the binding of the clathrin adaptor AP-2 and the endocytosis of the NR2B-containing NMDARs (Prybylowski et al., 2005). PSD-95 also binds Src (Kalia and Salter, 2003; Kalia et al., 2006) and amino acids 1-54 of PSD-95 are sufficient for SRC-PSD-95 binding (Kalia et al., 2006). Cdk5 phosphorylates the region of the $\mathrm{N}$ terminus of PSD-95 that binds Src (Morabito et al., 2004). Therefore, we hypothesized that Cdk5 regulates the binding of Src to PSD-95 and the Src-dependent phosphorylation of Y1472 NR2B thereby regulating the surface expression of NR2B-containing NMDARs.

To assess the role of Cdk5 in the phosphorylation of Y1472 NR2B in neurons, dissociated cultured cortical neurons were treated for 45 min with DMSO (control) or $5 \mu \mathrm{M}$ roscovitine, a specific inhibitor of $\mathrm{Cdk} 5$, a treatment that results in a decrease in Cdk5 activity as detected by a decrease in phosphorylation of tyrosine 15 Cdk5 (Fu et al., 2007). NR2B immunoprecipitated from lysates of these cultured neurons was analyzed with a phospho-Y1472 NR2B-specific antibody. Compared with the control, cultures treated with roscovitine revealed a sixfold increase in Y1472 NR2B phosphorylation $(685.55 \pm 175.69 \%$ of control) (Fig. 1A). To further evaluate the impact of Cdk5 activity on the phosphorylation of Y1472, we analyzed lysates derived from Cdk5-/- brains of E17.5 mouse embryos by immunoblotting. Comparison with the control littermates $(\mathrm{Cdk} 5+/-)$ revealed an increase in Y1472 NR2B phosphorylation in the Cdk5-/ - lysates (706.88 $\pm 62.65 \%$ of control), consistent with the increased phosphorylation of Y1472 NR2B observed in neurons treated with roscovitine (Fig. $1 B$ ). This indicated that the level of phosphorylation of Y1472 NR2B is dependent on the activity of Cdk5. To determine whether the increase in phosphorylation of Y1472 NR2B observed when Cdk5 is inhibited is dependent on SFKs, we treated the neuronal cultures with $1 \mu \mathrm{M}$ SU6656, a specific inhibitor of SFKs, and $5 \mu \mathrm{M}$ roscovitine for 45 min. The phosphorylation of Y1472 NR2B was substantially de-

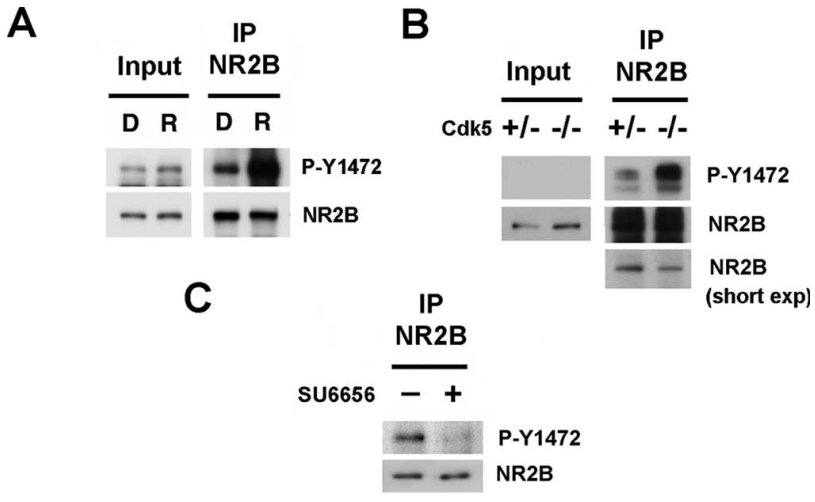

Figure 1. Inhibition of $C d k 5$ in neurons results in increased phosphorylation of NR2B at residue Y1472. $A$, The level of phosphorylation of NR2B at residue Y1472 was monitored in neuronal cortical cultures treated with roscovitine ( $5 \mu \mathrm{M}$ ) or DMSO (as control) for $45 \mathrm{~min}$. Immunoprecipitated (IP) NR2B from lysates of cultures treated with roscovitine (R) or DMSO (D) were analyzed with phospho-Y1472 NR2B-specific antibody. Quantification shows that roscovitine treatment increases the phosphorylation of $Y 1472$ NR2B compared with control $(685.55 \pm 175.69 \% ; p=0.098 ; n=5) . B$, The phosphorylation of NR2B at residue $Y 1472$ was analyzed in Cdk5 $-/-$ and Cdk5 $+/-$ brains from E17.5 mice. NR2B immunoprecipitated from brain lysates was analyzed with phospho-Y1472 NR2B-specific antibody. Phosphorylation of Y1472 NR2B increased in Cdk5 - / - compared with Cdk5 + / - brains a (706.885 \pm $62.65 \%$ of control; $p=0.222 ; n=2)$. exp, Exposure. $C$, The level of phosphorylation of NR2B at residue $\mathrm{Y} 1472$ was monitored in neuronal cortical cultures treated for 45 min with roscovitine ( 5 $\mu \mathrm{M}$ ) alone or with roscovitine and SU6656, an inhibitor of SFKs. NR2B immunoprecipitated from the lysates of the treated cultures was analyzed with phospho-Y1472 NR2B-specific antibody. There was a decrease in phospho-Y1472 NR2B in cultures treated with SU6656 compared with cultures not treated with SU6656 (46.74 $\pm 6.44 \%$ of control; $p=0.245 ; n=2)$.

creased by SU6656 treatment $(46.74 \pm 6.44 \%$ of control), indicating that the observed increase in phosphorylation of NR2B on Y1472 is SFKs-dependent (Fig. $1 C$ ). Together these results indicate that the SFKs-dependent phosphorylation of NR2B on Y1472 is increased when Cdk5 is inhibited.

\section{Src phosphorylates Y1472 NR2B in a Cdk5- and PSD-95-dependent manner}

Among the SFKs, Src plays a central role in the regulation of NMDARs, but the molecular mechanisms by which Src asserts its function are not clear. To determine whether, in the presence of PSD-95, Src phosphorylates Y1472 NR2B in a Cdk5-dependent manner, we analyzed the phosphorylation level of Y1472 NR2B in lysates of HEK 293Tcells expressing a constitutively active mutant of chick Src (Y527F), PSD-95, NMDARs subunits NR1 and NR2B, and either p35-Cdk5 (active) or p35-DNk5 (dominant negative Cdk5, inactive). Phosphorylation of Y1472 NR2B is significantly increased in lysates expressing p35-DNk5 compared with those expressing p35-Cdk5 (257.17 $\pm 56.23 \%$ and $126.33 \pm$ $30.92 \%$ respectively; $p=0.022$ ) (Fig. $2 A$ ). Previous studies have implicated Fyn in the phosphorylation of Y1472 NR2B, suggesting that Fyn may also regulate the phosphorylation of Y1472 NR2B in a Cdk5-dependent manner. To investigate this possibility, we analyzed the phosphorylation level of Y1472 NR2B in lysates of HEK 293Tcells expressing a constitutively active mutant of Fyn (Y531F), PSD-95, NMDARs subunits NR1 and NR2B, and either p35-Cdk5 (active) or p35-DNk5 (dominant negative Cdk5, inactive). In contrast to Src, Fyn phosphorylation of Y1472 NR2B is not increased in lysates expressing p35-DNk5 compared with those expressing p35-Cdk5 (121.65 $\pm 44.78 \%$ and $170.67 \pm$ $53.42 \%$, respectively) (Fig. $2 \mathrm{~B}$ ), consistent with a role for Src (and not Fyn) in the phosphorylation of Y1472 NR2B as Cdk5 is inhibited. 
A

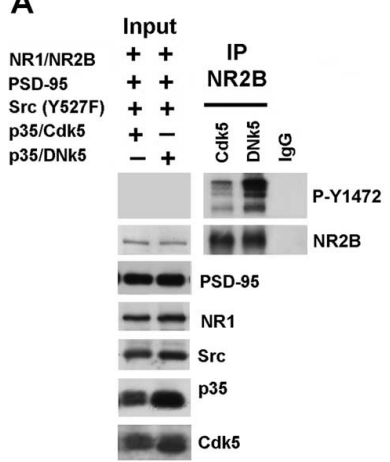

B

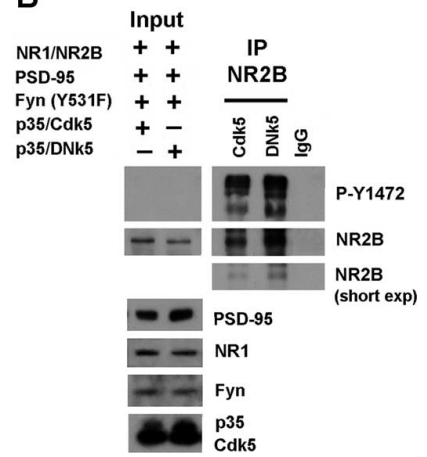

Figure 2. Src phosphorylates NR2B on Y1472 in a Cdk5-dependent manner. A, The level of phosphorylation of NR2B at residue Y1472 was monitored in transfected HEK 293T cells expressing NR1/ NR2B, PSD-95, Y527F Src (active), and either p35-cdk5 (active) or p35-DNk5 (inactive). NR2B was immunoprecipitated (IP) from thelysates of transfected cultures and probed with the phospho-Y1472 NR2B-specific antibody. Quantification shows a significant increase in Y1472 NR2B phosphorylation when dominant negative, inactive $C \mathrm{dk} 5$ ( $\mathrm{p} 35$-DNk5) was expressed compared with active $C \mathrm{dk} 5$ ( $\mathrm{p} 35$ cdk5) $(257.17 \pm 56.23 \%$ and $126.33 \pm 30.92 \%$, respectively; $p=0.022 ; n=6)$. $\boldsymbol{B}$, The level of phosphorylation of NR2B at residue Y1472 was monitored in transfected HEK 293T cells expressing a constitutively active mutant of Fyn (Y531F), PSD-95, NMDARs subunits NR1 and NR2B, and either p35-Cdk5 (active) or p35-DNk5 (inactive). Quantification of the level of phospho-Y1472 of immunoprecipitated NR2B reveals that Fyn phosphorylation of Y1472 NR2B is not increased in lysates expressing p35-DNk5 compared with those expressing p35-Cdk5 (121.65 $\pm 44.78 \%$ and $170.67 \pm 53.42 \%$, respectively; $p=0.194 ; n=5)$. exp, Exposure.
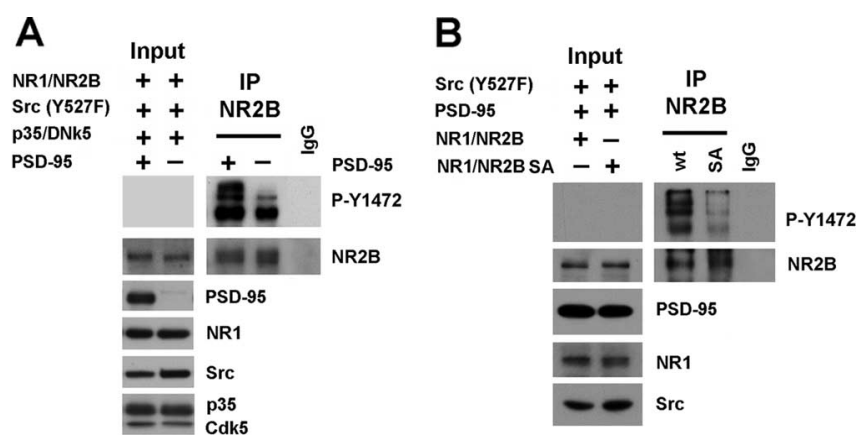

Figure 3. PSD-95 regulates the phosphorylation of Y1472 NR2B by Src. $A$, The impact of PSD-95 expression on the Src-dependent phosphorylation of Y1472 NR2B was analyzed in transfected HEK 293T cells expressing NR1/NR2B, Y527F Src (active), p35-DNk5 (inactive), with or without PSD-95. Immunoprecipitated (IP) NR2B was immunoblotted with the phosphoY1472 NR2B-specific antibody. Quantification shows that the expression of PSD-95 increases significantly the Src-dependent phosphorylation of Y1472 NR2B by nearly fourfold (226.246 \pm $41.98 \%$ compared with $60.65 \pm 20.39 \%$ in lysates without PSD-95; $p=0.027 ; n=5$ ). $\boldsymbol{B}$, The phosphorylation of Y1472 NR2B by Src was analyzed in HEK 293T cells expressing Src (Y527F), PSD-95, NR1, and either Flag-NR2B or Flag-NR2B S1480A, a mutation that dramatically decreases the binding of NR2B to the PDZ domains of PSD-95. Expression of the S1480A NR2B mutant $(S A)$ resulted in a decrease in phosphorylation of $\mathrm{Y} 1472(41.31 \pm 10.76 \%$ vs $97.99 \pm$ $35.60 \%$ of wild-type NR2B; $p=0.142 ; n=4$ ). Wt, Wild type.

PSD-95 binds NR2B at synapses and also binds Src, therefore it may influence the phosphorylation of Y1472 NR2B by Src. To analyze whether PSD-95 plays a role in the phosphorylation of Y1472 NR2B by Src, we expressed Src (Y527F), NR1, NR2B, and p35-DNk5, either with or without PSD-95 in HEK 293Tcells. Expression of PSD-95 resulted in a nearly fourfold significant increase in phosphorylation of Y1472 NR2B (226.246 $\pm 41.98 \%$ versus $60.65 \pm 20.39 \%$ in lysates without PSD-95; $p=0.027$ ) (Fig. 3A). To further analyze whether the phosphorylation of Y1472 NR2B by Src is dependent on the binding of NMDARs to PSD-95, we expressed Src (Y527F), PSD-95, NR1, and either Flag-NR2B or Flag-NR2B S1480A, a mutation that dramatically
A

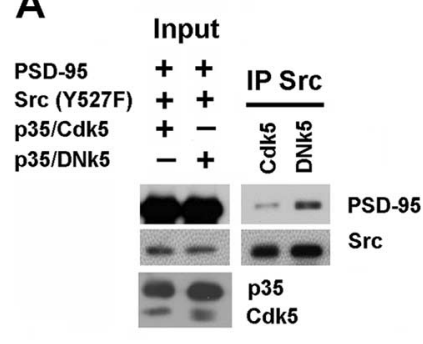

B

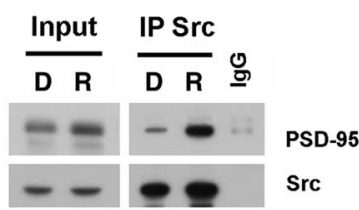

C

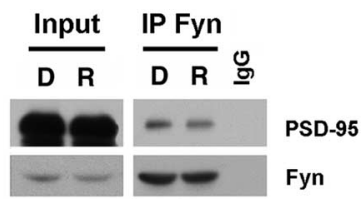

Figure 4. Cdk5 activity regulates the binding of PSD-95 to Src but not Fyn. $A$, The impact of Cdk5 activity on the binding of PSD-95 to Src was evaluated in transfected HEK 293T cells expressing PSD-95, Y527F Src (active), and either p35-Cdk5 (active) or P35-DNk5 (inactive). Src was immunoprecipitated (IP) from the lysates of transfected cultures and analyzed for coimmunoprecipitation of PSD-95. Quantification shows a significant increased interaction between Src and PSD-95 after expression of inactive Cdk5 (810.53 $\pm 289.8 \%$ of active $C d k 5, p=0.002$; $n=5$ ). $\boldsymbol{B}$, The level of binding of PSD-95 with Src was monitored in neuronal cortical cultures treated with roscovitine $(5 \mu \mathrm{M}$ ) or DMSO (as control) for $45 \mathrm{~min}$. Src immunoprecipitated from lysates of cultures treated with roscovitine (R) or DMSO (D) was analyzed for the amount of PSD-95 coimmunoprecipitated with Src. Quantification shows a significant increased interaction between $\mathrm{Src}$ and PSD-95 in roscovitine-treated neurons ( $378.59 \pm 59.25 \%$ of DMSO control, $p=0.0083 ; n=6$ ). C, The level of binding of PSD-95 with Fyn was monitored in neuronal cortical cultures treated with roscovitine $(5 \mu \mathrm{M}$ ) or DMSO (as control) for $45 \mathrm{~min}$. Fyn immunoprecipitated from lysates of cultures treated with roscovitine (R) or DMSO (D) was analyzed for the amount of PSD-95 coimmunoprecipitated. Quantification shows that roscovitine treatment does not alter significantly the binding of Fyn to PSD-95 (95.33 $\pm 12.66 \%$ of DMSO control, $p=0.973 ; n=4)$.

decreases the binding of NR2B to the PDZ domains of PSD-95 (Lim et al., 2002; Prybylowski et al., 2005), in HEK 293T cells. Expression of the S1480A NR2B mutant resulted in a decrease in phosphorylation of Y1472 NR2B (41.31 $\pm 10.76 \%$ vs $97.99 \pm$ $35.60 \%$ of wild-type NR2B) (Fig. $3 B$ ). Together, these experiments indicate that Src phosphorylates Y1472 NR2B in a Cdk5and PSD-95-dependent manner, suggesting that PSD-95, by binding to Src and NR2B, facilitates the phosphorylation of Y1472 NR2B by Src.

\section{Cdk5 inhibition increases the interaction of PSD-95 with Src} but not Fyn

The region of the $\mathrm{N}$ terminus of PSD-95 that binds Src (Kalia et al., 2006) is also phosphorylated by Cdk5 (Morabito et al., 2004). Therefore, we next examined whether Cdk5 activity regulated the binding of Src to PSD-95 by analyzing this interaction in transfected HEK 293T cells that expressed active Src, PSD-95, and either p35-Cdk5 or p35-DNk5. Although a weak interaction was detected between Src and PSD-95 in cells expressing p35-Cdk5, this interaction increased significantly with expression of $\mathrm{p} 35$ DNk5 (810.53 $\pm 289.80 \%$ of active Cdk5, $p=0.002)$ (Fig. $4 A)$. To confirm in neurons the role of Cdk5 in the regulation of the interaction of Src with PSD-95, we treated cultured cortical neurons for $45 \mathrm{~min}$ with $5 \mu \mathrm{M}$ roscovitine, or DMSO as control. Consistent with the observations in HEK 293T cells, there was approximately a fourfold significant increase in Src binding to PSD-95 after roscovitine treatment $(378.59 \pm 59.25 \%$ of DMSO control, $p=0.0083$ ) (Fig. $4 \mathrm{~B}$ ), indicating that, in neurons as well as in transfected cells, the binding of Src with PSD-95 increased when $\mathrm{Cdk} 5$ was inhibited. Whereas Src binds to the $\mathrm{N}$ terminus of PSD-95, Fyn binds to the PDZ3 region of PSD-95 (Tezuka et al., 1999), suggesting distinct regulatory mechanisms by which Src and Fyn bind to PSD-95. To test whether in neurons the binding of Fyn to PSD-95 is independent of Cdk5 activity, we treated 

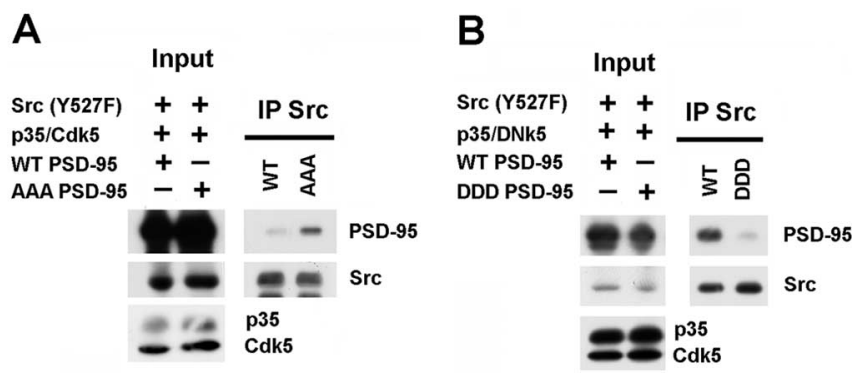

Figure 5. Phosphorylation of PSD-95 by Cdk5 decreases the binding of Src to PSD-95. $A$, The impact of the phosphorylation of the $\mathrm{N}$ terminus of PSD-95 by Cdk5 on the binding of PSD-95 to Src was evaluated in transfected HEK 293T cells expressing Y527F Src (active), p35-Cdk5 (active), and either wild-type PSD-95 or the alanine mutant of PSD-95 (T19A, S25A, S35A; AAA PSD-95). Src immunoprecipitated from lysates of transfected cultures was analyzed for the amount of AAA PSD-95 coimmunoprecipitated with Src. Quantification shows that Src binds significantly more to AAA PSD-95 compared with wild-type PSD-95 (786.33 $\pm 156 \%$ of wildtype, $p=0.001 ; n=4)$. $B$, The impact of the phosphorylation of the N terminus of PSD-95 by Cdk5 on the binding of PSD-95 to Src was evaluated in transfected HEK 293T cells expressing Y527F Src (active), p35-DNk5 (inactive), and either wild-type PSD-95 or the phosphorylationmimic of PSD-95 (T19D, S25D, S35D; DDD PSD-95). Src immunoprecipitated from lysates of transfected cultures was analyzed for the amount of DDD PSD-95 coimmunoprecipitated with Src. Quantification shows that Src binds less to DDD PSD-95 compared with wild-type PSD-95 $(38.28 \pm 19.01 \%$ of wild-type; $p=0.162 ; n=3)$.

cultured cortical neurons for 45 min with $5 \mu \mathrm{M}$ roscovitine, or DMSO as control. These neurons did not show a significant change in the binding of Fyn to PSD-95 (95.33 $\pm 12.66 \%$ of DMSO control; $p=0.973$ ) (Fig. $4 C$ ). Thus, inhibition of Cdk5 results in a statistically significant increase in the binding of PSD-95 to Src but not Fyn.

Next, we examined whether phosphorylation of the $\mathrm{N}$ terminus of PSD- 95 by Cdk 5 regulates the binding of Src to PSD-95 by expressing the Cdk5-phosphorylation mutants of PSD-95 in HEK 293T cells: the alanine mutant [T19A, S25A, S35A (AAA PSD-95)] (Morabito et al., 2004) and the phosphomimic mutant [T19D, S25D, S35D (DDD PSD-95)]. Indeed, we observed significantly increased binding of Src to AAA PSD-95 compared with wild-type PSD-95 (786.33 $\pm 156 \%$ of wild-type; $p=0.001)$ in transfected HEK 293T cells expressing constitutively active Src (Y527F), p35-Cdk5 and either AAA PSD-95 or wild-type PSD-95 (Fig. 5A). This result is consistent with the increased binding of Src with PSD-95 under Cdk5 inhibition. Conversely, there was less binding of Src with DDD PSD-95 than with wild-type PSD-95 (38.28 $\pm 19.01 \%$ of wild-type) in transfected HEK 293T cells expressing active Src, p35-DNk5 and either DDD PSD-95 or wild-type PSD-95 (Fig. 5B). Together these observations indicated that Cdk5-dependent phosphorylation of the $\mathrm{N}$ terminus of PSD-95 regulates the binding of Src with PSD-95.

\section{Cdk5 inhibition increases the activity of Src and the phosphorylation of Y1472 NR2B at synapses}

At synapses, Fyn phosphorylation of NR2B has provided a mechanism for rapid modulation of NR2B-containing NMDARs, although NMDARs are also localized at extrasynaptic sites, where they play a role in excitotoxicity (Hardingham and Bading, 2003). Both Cdk5 and Src are also present at synapses, suggesting that Src may contribute to the phosphorylation of synaptic NR2B. To address whether Y1472 NR2B is phosphorylated at synapses in a Cdk5-dependent manner, we analyzed the levels of phosphorylation of Y1472 NR2B in synaptosomal membrane fractions derived from acute forebrain slices of adult mouse after treatment with $15 \mu \mathrm{M}$ roscovitine for $45 \mathrm{~min}$, or DMSO as control. Lysis of
A

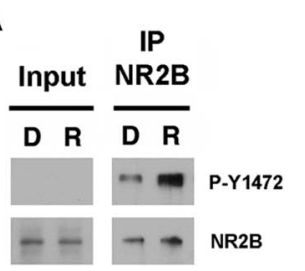

B

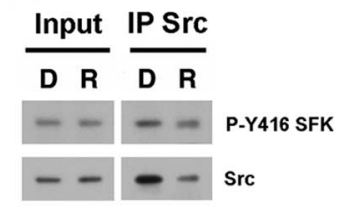

Figure 6. Cdk5 inhibition increases the activity of Src and the phosphorylation of residue Y1472 NR2B at synapses. $\boldsymbol{A}$, The level of phosphorylation of NR2B at residue Y1472 was monitored in synaptosomal membrane fractions derived from acute forebrain slices of adult mouse treated with $15 \mu \mathrm{m}$ roscovitine (R) for $45 \mathrm{~min}$, or DMSO (D) as a control. NR2B immunoprecipitated from synaptosomal membrane fractions of forebrain slices was analyzed with the phospho-Y1472 NR2B-specific antibody. Quantification shows that roscovitine treatment increases the phosphorylation of Y1472 NR2B compared with control DMSO (387.55 $\pm 181.71 \%$ of control; $p=0.277 ; n=3$ ). $\boldsymbol{B}$, The phosphorylation levels of Y416, a marker of active SFKs, was analyzed in synaptosomal membrane fractions derived from acute forebrain slices of adult mouse treated with $15 \mu \mathrm{m}$ roscovitine (R) for $45 \mathrm{~min}$, or DMSO (D) as a control. Src immunoprecipitated from synaptosomal membrane fractions of forebrain slices treated with roscovitine or DMSO was analyzed with the phospho-Y416 SFK specific antibody. Quantification and normalization to the amount of Src immunoprecipitated reveals that roscovitine treatment significantly increases approximately twofold the phosphorylation of Y416 Src compared with control $(225.48 \pm 54.80 \%$ of DMSO control; $p=0.033 ; n=3)$

the synaptosomal membrane fractions was followed by immunoprecipitation of NR2B and immunoblotting with the phosphoY1472 NR2B-specific antibody. We observed an increase in the phosphorylation of Y1472 NR2B after roscovitine treatment (387.55 $\pm 181.71 \%$ of DMSO control), consistent with the SFKCdk5-dependent phosphorylation of synaptic NMDARs (Fig. $6 A$ ). The phosphorylation of NR2B by Src would require the presence of active Src in the synaptosomal membrane fractions and, because inhibition of Cdk5 resulted in increased phosphorylation of NR2B, it is possible that Cdk5 affects Src activity, as suggested by previous studies (Gao et al., 2004). Src activity is regulated by tyrosine phosphorylation at Tyr 416 (Y416) in the activation loop of the kinase domain (Hunter, 1987). To analyze whether at synapses Src activity is regulated by Cdk5, we monitored Cdk5-dependent changes in Src activity in synaptosomal membrane fractions derived from acute forebrain slices of adult mouse after treatment with $15 \mu \mathrm{M}$ roscovitine for $45 \mathrm{~min}$, or DMSO as control. Immunoprecipitation of Src from these fractions, immunoblotting with phospho-Y416 SFK antibody, and normalization to the amount of Src immunoprecipitated, revealed that phosphorylation of Y416 Src is significantly increased by approximately twofold in the fractions derived from roscovitine-treated forebrain slices $(225.48 \pm 54.80 \%$ of DMSO control; $p=0.033$ ) (Fig. $6 B$ ). Together, these results support the model in which inhibition of Cdk5 results in increased activation of Src and that, in turn, Src phosphorylates Y1472 NR2B at synaptic membranes in a Cdk5-dependent manner.

Cdk5 inhibition decreases the association of $\boldsymbol{\beta} 2$-adaptin with NR2B and the activity-dependent internalization of NR2B/NMDARs

The YEKL endocytic motif of NR2B binds the AP-2 adaptor complex and thus links NR2B to clathrin (Lavezzari et al., 2003). Phosphorylation of Y1472 NR2B within the YEKL motif blocks the binding of AP-2 to NR2B and increases the surface expression of NR2B-containing NMDARs (Prybylowski et al., 2005; Snyder et al., 2005). Because inhibition of Cdk5 resulted in increased Y1472 NR2B phosphorylation, we predicted that inhibition of Cdk5 should result in decreased binding of AP-2 to NR2B, leading to decreased activity-dependent NMDARs endocytosis. To 
investigate whether Cdk5 positively regulates the association of NR2B with AP-2 in neurons, we pretreated cultured cortical neurons with $5 \mu \mathrm{M}$ roscovitine (or DMSO) for $45 \mathrm{~min}$ followed by treatment (in the continuous presence of roscovitine or DMSO) with $100 \mu \mathrm{M}$ glycine for $5 \mathrm{~min}$ to prime NMDARs for endocytosis (Nong et al., 2003). As predicted, $\beta 2$-adaptin, a component of the AP-2 complex, was significantly less associated with NR2B in neurons treated with roscovitine (42.76 士 $13.81 \%$ of control; $p=0.01$ ) (Fig. $7 A$ ). These observations suggest that stimulation of the extracellular glycine primes the NMDAR for internalization by allowing the interaction of NMDARs with $\beta 2$ adaptin/AP-2 and the endocytic machinery, whereas the addition of NMDA allows the NMDAR internalization to proceed. Because inhibition of Cdk5 resulted in reduced binding of AP-2 to NR2B, it should prevent the activity-dependent endocytosis of NR2B-containing NMDARs.

To assess the effect of Cdk5 inhibition on NMDAR internalization, we analyzed the level of surface expression of NR2B after glycine/NMDA stimulation (Nong et al., 2003) of cortical neurons treated with roscovitine or DMSO. Previous studies have shown that in neurons, the extracellular region of NMDARs is cleaved by the extracellular protease chymotrypsin and the decrease in the NR2B immunoblot signal is proportional to NR2B/NMDARs endocytosis (Hall and Soderling, 1997; Fong et al., 2002). Therefore, cortical neuronal cultures pretreated with $5 \mu \mathrm{M}$ roscovitine (or DMSO as control) for $45 \mathrm{~min}$ were primed with $100 \mu \mathrm{M}$ glycine for $5 \mathrm{~min}$, followed by stimulation with $100 \mu \mathrm{M}$ glycine, $50 \mu \mathrm{M}$ NMDA for $5 \mathrm{~min}$, in the constant presence of $5 \mu \mathrm{M}$ roscovitine or DMSO. After treatment, live neurons were exposed to chymotrypsin $(0.05 \mathrm{mg} / \mathrm{ml}$ for $10 \mathrm{~min})$ to determine the surface expression of NR2B. The intracellular protein actin is inaccessible to chymotrypsin and was used as a loading control. Under control conditions (DMSO), after stimulation with glycine/NMDA, $89.19 \pm 5.14 \%$ of NR2B immunoreactivity was maintained. Because internalization preserves the NR2B epitope, this result indicates decreased surface expression of NR2B in control cultures. In contrast, in neurons treated with roscovitine, only $47.05 \pm 6.96 \%$ of NR2B immunoreactivity remained. Thus, inhibition of Cdk5 activity resulted in a statistically significant $(p=0.002)$ reduction in the internalization of NR2B-containing NMDARs compared with the control (Fig. $7 B)$.

We also analyzed by immunocytochemistry whether Cdk5 inhibition reduces the activity-dependent internalization of NR2B-containing NMDARs (Fig. 7C,D). Cultured cortical neurons were treated with roscovitine (or DMSO) and glycine/ NMDA as described above. After treatments, neurons were fixed
B

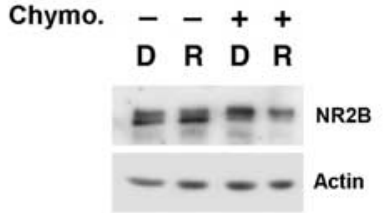

D

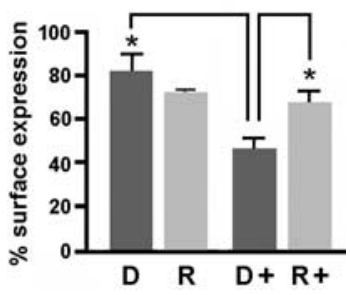

Figure 7. Inhibition of C $\mathrm{dk} 5$ in neurons decreases the binding of NR2B with the AP- 2 complex and the endocytosis of NMDARs. $A$, The level of binding of NR2B with the AP- 2 complex was monitored in neuronal cortical cultures treated with roscovitine $(5 \mu \mathrm{M})$ or DMSO (as control) for $45 \mathrm{~min}$ and with roscovitine (or DMSO) and $100 \mu \mathrm{m}$ glycine for $5 \mathrm{~min}$. NR2B immunoprecipitated from 列 there is a significant decrease in the binding of NR2B with $\beta 2$-adaptin in cultures treated with roscovitine ( $42.76 \pm 13.81 \%$ of 作, $p=0.01 ; n=5$ ). $\boldsymbol{B}$, The levels of surface expression of NR2B were evaluated in neuronal cortical cultures treated with by additional 5 min in $100 \mu \mathrm{m}$ glycine and $50 \mu \mathrm{m} \mathrm{NMDA}$ (with roscovitine or DMSO). Live neuronal cultures were then treated with $0.05 \mathrm{mg} / \mathrm{ml}$ chymotrypsin for $10 \mathrm{~min}$ at $37^{\circ} \mathrm{C}$ to cleave the extracellular domain of the NMDARs on the membrane surface $(D+, R+)$ or left untreated $(D-, R-)$. The lysates derived from these neuronal cultures were analyzed for the expression of uncleaved NR2B and actin (as loading control). Chymo, Chymotrypsin. Quantification indicates that, under control conditions, ivity is present $(p=0.002 ; n=3)$. C, The levels of surface expression of NR2B were evaluated in neuronal cortical cultures -i) 5 before being permeabilized $(\boldsymbol{a}, \boldsymbol{d}, \boldsymbol{g}, \boldsymbol{j})$ and immunostained for NR2B after permeabilization to visualize all NR2B-containing NMDARs $(\boldsymbol{b}, \boldsymbol{e}, \boldsymbol{h}, \boldsymbol{k})$. Overlays of NR1 and NR2B immunostaining $(\boldsymbol{c}, \boldsymbol{f}, \boldsymbol{i}, \boldsymbol{I})$. $\boldsymbol{D}$, Quantification of staining conducted on coded material using MetaMorph software. In naive control cultures (D, $C a-C c), 81.50 \pm 7.30 \%$ of NR2B-containg NMDARs are present $6.65 \pm 1.85 \%$ and $75.32 \pm 1.92 \%$, respectively; $\left.F_{(2.12)}=12.3 ; p<0.001\right)$ and the main effect of glycine-NMDA stimulation stimulation, $F=26.16, p<0.0001)$. Post hoc pairwise comparisons were evaluated using the Tukey's HSD test. Statistically ( $p<0.05)$ differences were found between the average number of puncta of the DMS0-glycine/NMDA (D+) and the DMSO nonstimulated (D) groups and between the average number of puncta of the DMSO-glycine/NMDA (D+) and the roscovitine-glycine/NMDA ( $R+$ ) groups (D). Total puncta: $D, 391 ; D+, 500 ; R, 350 ; R+, 366$. Error bars indicate SEM.

without permeabilization, and immunostained with an antibody to NR1 that recognizes an extracellular epitope. The degree of permeabilization in the absence of Triton X-100 was monitored by staining for intracellular synaptic proteins such as PSD-95, which remained basically undetactable (data not shown). The NR1 immunostaining was followed by permeabilization of neurons and immunostaining with an antibody to NR2B to visualize both surface and intracellular NR2B-containing NMDARs. In naive control cultures (DMSO, not stimulated), $81.50 \pm 7.30 \%$ of NR2B puncta were also stained with NR1 antibody, whereas after treatment of control cultures with glycine/NMDA, only $46.85 \pm 4.61 \%$ ) of NR2B puncta were also positive for NR1. This decrease is consistent with the observation that glycine/NMDA treatment reduces the surface expression of NMDARs. In contrast, in cultures treated with roscovitine, $73.79 \pm 0.51 \% \mathrm{NR} 2 \mathrm{~B}$ 
puncta were positive for NR1 and, after stimulation with glycine/ NMDA, the number of NR2B puncta positive for NR1 was comparable with that of unstimulated cultures $(67.14 \pm 5.17 \%)$, but statistically different from that of stimulated control cultures $(46.85 \pm 4.61 \%)$. A two-way ANOVA was performed to statistically compare the average number of puncta in the four experimental conditions: DMSO (with and without glycine-NMDA stimulation) and roscovitine (with and without glycine-NMDA stimulation). The overall effect was statistically significant $\left(F_{(3,21)}\right.$ $=23.13 ; p<0.0001)$, as also were the overall effect of roscovitine versus DMSO $(86.65 \pm 1.85 \%$ and $75.32 \pm 1.92 \%$, respectively; $\left.F_{(2,12)}=12.3, p<0.001\right)$ and the main effect of glycine-NMDA stimulation (with, $72.32 \pm 1.84 \%$ vs without, $87.65 \pm 1.91 \%$; $F_{(2,12)}=33.1, p<0.0001$ ) and the interaction (roscovitine by glycine-NMDA stimulation, $F=26.16, p<0.0001$ ). Post hoc pairwise comparisons were evaluated using the Tukey's HSD test. Statistically significant $(p<0.05)$ differences were found between the average number of puncta of the DMSO-glycine/ NMDA $(\mathrm{D}+)$ and the DMSO nonstimulated (D) groups and between the average number of puncta of the DMSO-glycine/ NMDA $(\mathrm{D}+)$ and the roscovitine-glycine/NMDA $(\mathrm{R}+)$ groups (Fig. 7D). Therefore, whereas in control cultures the surface expression of NR2B-containing NMDARs is decreased significantly by glycine/NMDA stimulation, in roscovitine-treated cultures there was not a statistically significant change in surface expression of NMDARs. Together these studies indicate that Cdk5 regulates the activity-dependent surface expression of NR2Bcontaining NMDARs.

\section{Discussion}

The surface expression of NMDARs is dynamic and their trafficking, insertion, and internalization is tightly regulated. Both the constitutive and activity-dependent internalization of NMDARs depend on clathrin-dependent endocytosis. Our study demonstrates that inhibition of Cdk5, a kinase implicated in synaptic plasticity, inhibits the endocytosis and increases the surface expression of NMDARs by regulating the phosphorylation of NR2B on residue Y1472, which decreases the association of NR2B with $\beta 2$-adaptin, a component of the clathrin adaptor protein complex AP-2. Based on our data, we propose a model in which inhibition of Cdk5 results in phosphorylation of Y1472 NR2B by Src, which is facilitated by the increased binding of Src to PSD-95. In turn, this phosphorylation reduces the binding of phosphoY1472 NR2B to AP-2 and thus reduces the activity-dependent internalization of NMDARs.

Activity of NMDARs is positively regulated by SFKsdependent tyrosine phosphorylation (Wang and Salter, 1994; Kohr and Seeburg, 1996; Yu et al., 1997). NR2B is a substrate of SFKs (Salter and Kalia, 2004) and the phosphorylation of NR2B at Y1472 positively correlates with NMDA currents (Alvestad et al., 2003) and with surface expression of NMDARs (Prybylowski et al., 2005; Snyder et al., 2005). We found that phosphorylation of Y1472 NR2B is increased when Cdk5 activity is pharmacologically inhibited by roscovitine treatment or is absent, such as in brain lysates of Cdk5-/- embryos. The observed Cdk5dependent increase in Y1472 NR2B phosphorylation is likely caused by the activity of SFKs, as suggested by the decrease in Y1472 NR2B phosphorylation observed when neurons were treated with the SFKs inhibitor SU6656. Although Fyn has been identified as the predominant kinase responsible for the phosphorylation of Y1472 NR2B in HEK 293T transfected cells (Nakazawa et al., 2001), in Fyn mutant mice the Y1472 NR2B phosphorylation is reduced, but not abolished (Nakazawa et al., 2001), suggesting that SFKs other than Fyn can phosphorylate Y1472 NR2B. Consistent with this possibility, we showed that, in transfected HEK 293T cells, the level of phosphorylation of Y1472 NR2B by Src increased substantially when Cdk 5 is inactivated, whereas this increase is not observed in HEK 293T cells transfected with constitutively active Fyn. This observation indicates that Src, rather than Fyn, phosphorylates Y1472 NR2B in a Cdk5dependent manner and suggests that Src may be responsible for the residual phosphorylation of Y1472 NR2B in Fyn - / - mice.

PSD-95 promotes Fyn-mediated phosphorylation of NMDARs (Tezuka et al., 1999), suggesting that PSD-95 could also facilitate the phosphorylation of NR2B by Src. In fact, our study shows that expression of PSD-95 in HEK 293T cells increases more than threefold the phosphorylation of Y1472 NR2B by Src, and that the expression of NR2B S1480A, a mutation that dramatically decreases the binding of NR2B to PSD-95, results in a decrease in phosphorylation of Y1472 NR2B. These results are consistent with a scaffolding role for PSD-95 in the Src-mediated phosphorylation of NR2B by PSD-95. Whereas Fyn binds the PDZ3 domain of PSD-95, Src binds the SH3-domain and the N terminus of PSD-95 (Kalia and Salter, 2003; Kalia et al., 2006), although the $\mathrm{N}$ terminus of PSD-95 is sufficient for binding with Src (Kalia et al., 2006) and is phosphorylated by Cdk5 (Morabito et al., 2004). Our study demonstrates that Cdk5 activity regulates the interaction of Src with PSD-95 both in transfected HEK 293T cells and in cortical neurons, whereas, in neurons, Fyn binds PSD-95 independently of Cdk5 activity. We also show that Src binds more to the alanine mutant of Cdk5 phosphorylation sites (T19A, S25A, S35A) in the N terminus of PSD-95 than to the phosphomimic mutant (T19D, S25D, S35D) of PSD-95, thus providing evidence that the phosphorylation of the $\mathrm{N}$ terminus of PSD-95 by Cdk5 regulates the Cdk5-dependent binding of Src to PSD-95. It has been shown previously that the binding of PSD-95 to the SH2 domain of Fyn promotes tyrosine phosphorylation of the NMDAR subunit NR2A (Tezuka et al., 1999), although, recently, Kalia et al. (2006) have proposed that the binding of PSD-95 to the SH2 domain of Src inhibits Src activity. In support of this conclusion, they show that in HEK 293 cells, tyrosine phosphorylation of NR2B is enhanced when a deletion mutant (aa14-54) of the N terminus of PSD-95 (lacking the Cdk5 phosphorylation sites) is expressed together with Src (Kalia et al., 2006). Src could still bind the SH3 domain of the deletion mutant of PSD-95 (Kalia et al., 2003) and promote the phosphorylation of NR2B observed in this study. Although our study does not address this point directly, we show that expression of wild-type PSD-95 increases the phosphorylation of Y1472 NR2B by Src in a Cdk5-dependent manner, consistent with PSD-95 acting as a Cdk5-dependent scaffold for Src.

The Cdk5 kinase activity is significantly and rapidly reduced by depolarization (Schuman and Murase, 2003) and NMDA stimulation (Wei et al., 2005), independently of p35 degradation (Wei et al., 2005), consistent with the fast kinetics of Cdk5 inactivation being relevant to the regulation of surface expression of NR2B-containing NMDARs. Based on our data, inhibition of $\mathrm{Cdk} 5$ promotes the tethering of Src to the $\mathrm{C}$ terminus of NR2B, thus promoting the subsequent phosphorylation of NR2B by Src. Interestingly, our study suggests that Src activity at synapses is upregulated by inhibition of Cdk5, further implicating Cdk5 in the signaling pathways modulating NMDAR activity such as the Src-dependent upregulation of NMDAR activity downstream of protein kinase C (Lu et al., 1999; Huang et al., 2001), EphB receptors (Takasu et al., 2002), integrin receptors (Lin et al., 2003), and reelin receptors (Chen et al., 2005). 
NMDARs undergo activity-dependent endocytosis (Roche et al., 2001; Snyder et al., 2001, 2005; Nong et al., 2003; Scott et al., 2004; Prybylowski et al., 2005). NR2A and NR2B contain different sorting motifs within their $\mathrm{C}$ terminus that regulate trafficking to distinct intracellular pathways after endocytosis (Lavezzari et al., 2004). In this study we have focused on the NR2B subunit, although it is possible that Cdk5 may also regulate the surface expression of NR2A, which is a direct substrate of Cdk5, likely through different mechanisms (Li et al., 2001). Phosphorylation of Y1472 NR2B downregulates the binding of NMDARs to the clathrin adaptor protein complex AP-2, and therefore, the interaction with the endocytosis machinery (Lavezzari et al., 2003). In neurons primed with glycine, the AP- 2 subunit $\beta 2$-adaptin associates with NR2B (Fong et al., 2002). Our study identifies a decrease in the association of $\beta 2$-adaptin with NR2B in neurons treated with roscovitine, consistent with the increase in phosphorylated Y1472 NR2B observed in these cultures. Furthermore, we show that when the endocytosis of NMDARs is stimulated by treatment with glycine/NMDA, roscovitine-treated neurons retain more NMDARs at the cell surface than control cultures. Therefore, this study identifies Cdk5 as a key component in the regulation of the activity-dependent internalization of NR2B-containing NMDARs. Although Cdk5 has been directly implicated in the mechanisms of clathrin-dependent endocytosis (Tan et al., 2003; Tomizawa et al., 2003; Lee et al., 2004, 2005), our data indicate a novel mechanism by which Cdk5 impacts the surface expression of NMDARs in an activity-dependent manner by regulating the level of phosphorylation of Y1472 NR2B.

The dynamic regulation of the surface expression of NMDARs is implicated in synapse maturation (Friedman et al., 2000; Washbourne et al., 2002; Bresler et al., 2004) and synaptic plasticity (Carroll and Zukin, 2002; Wenthold et al., 2003; PerezOtano and Ehlers, 2005). Tyrosine phosphorylation of NR2B is enhanced in LTP (Rosenblum et al., 1996; Rostas et al., 1996) and Y1472F NR2B mutant mice show impaired fear-related learning and reduced LTP in the amygdala, as well as NMDAR-mediated CaMKII signaling (Nakazawa et al., 2006). Importantly, Cdk5 has been implicated in LTP and LTD (Cheung et al., 2006) and conditional knock-out of Cdk5 in the adult mouse brain improved performance in spatial learning tasks and enhanced hippocampal LTP, NMDAR-mediated EPSCs, and surface expression of NR2B/NMDARs (Hawasli et al., 2007). These studies are consistent with our observations on the increase of Y1472 NR2B phosphorylation in roscovitine-treated neurons. Therefore, our study provides an additional molecular mechanism for the $1.9 \pm 0.2-$ fold increase in surface NR2B observed in brain slices of conditional knock-out of Cdk5 (Hawasli et al., 2007). Although this increase is not apparent in our dissociated cultures, a possible explanation is the relatively short time of inhibition by roscovitine compared with the longer absence of Cdk5 activity in the conditional knock-out to allow for such NR2B accumulation. Thus, our study provides a mechanism for the reduction of the threshold for LTP induction (Wei et al., 2005) and the impairment of LTD induction (Ohshima et al., 2005) observed in mice lacking active p35-Cdk5 (p35-/-) as well as for the enhanced synaptic plasticity and surface expression of NR2B/NMDARs observed in the conditional knock-out of Cdk5. Together these observations suggest that a molecular mechanism by which Cdk5 impacts NMDAR-dependent signaling and learning and memory is the Src-dependent phosphorylation of Y1472 NR2B.

Cdk5 regulates striatal dopamine signaling and changes associated with cocaine exposure (Bibb, 2003; Chergui et al., 2004; Taylor et al., 2007). Interestingly, increased tyrosine phosphory- lation and surface expression of NR2B-containing NMDARs have been associated with striatal dopamine D1 receptor activation (Dunah and Standaert, 2001; Hallett et al., 2006), and cocaine-induced behavioral sensitization in the nucleus accumbens (Zhang et al., 2007). Furthermore, Hallett et al. (2006) revealed that D1 receptor activation can potentiate striatal NMDA subunit function by directly promoting the surface insertion of the receptor complexes and that tyrosine phosphatase inhibition leads to the clustering of tyrosine-phosphorylated NR2B subunit along dendritic shafts. A possibility suggested by our study is that Cdk5 regulates NR2B trafficking as well as the D1 receptordependent regulation of NMDARs in striatum by regulating the phosphorylation of Y1472 NR2B, thus further implicating the deregulation of Cdk5 activity in the etiology of Parkinson's disease and drug addiction.

The Cdk5 activator p35 is cleaved by calpain and the calpainderived cleavage product p25 is implicated in neurotoxicity and neurodegeneration (Cruz and Tsai, 2004). There is increasing evidence of a physiological role for the formation of $\mathrm{p} 25$, possibly in synaptic plasticity (Fischer et al., 2005) and recent studies have shown that the bulk of p25 is formed primarily via NMDARmediated $\mathrm{Ca}^{2+}$ influx (Kerokoski et al., 2004). Thus, Cdk5 regulation of the surface expression of NR2B-containing NMDARs may provide a feedback regulatory loop, by regulating the amount of p 25 produced in spines and thus affecting synaptic plasticity and neurodegenerative disorders associated with Cdk5 activity. Moreover, analysis of an inducible p 25 transgenic mouse has shown that high level of p25 expression for 6 weeks results in impairment of LTP (Fischer et al., 2005). Based on our study, we speculate that prolonged elevated levels of p25-Cdk5 activity result in a loss of phosphorylated Y1472 NR2B and, consequently, in decreased surface expression of NMDARs, which in turn results in impaired NMDAR-dependent LTP.

Finally, addition of $A \beta$ to neurons results in the impairment of induction of LTP in CA1 that can be prevented by Cdk5 inhibitors (Wang et al., 2004), and in the endocytosis of NMDARs (Snyder et al., 2005). Our study indicates that Cdk5 inhibition regulates the surface expression of NMDARs by increasing the levels of phosphorylation of Y1472 NR2B, suggesting a mechanism by which Cdk 5 activity impacts the $A \beta$-dependent effect on NMDARs and the synaptic dysfunction observed in Alzheimer's (Selkoe, 2002; Lau and Zukin, 2007). Given the increasing implication of Cdk5 as a key regulator of synaptic function, our study contributes to the understanding of the biological role of Cdk5 at the synapse and the characterization of the signaling pathways involved in the regulation of NMDAR surface expression and function.

\section{References}

Ali DW, Salter MW (2001) NMDA receptor regulation by Src kinase signalling in excitatory synaptic transmission and plasticity. Curr Opin Neurobiol 11:336-342.

Alvestad RM, Grosshans DR, Coultrap SJ, Nakazawa T, Yamamoto T, Browning MD (2003) Tyrosine dephosphorylation and ethanol inhibition of N-Methyl-D-aspartate receptor function. J Biol Chem 278:11020-11025.

Barria A, Malinow R (2002) Subunit-specific NMDA receptor trafficking to synapses. Neuron 35:345-353.

Bibb JA (2003) Role of Cdk5 in neuronal signaling, plasticity, and drug abuse. Neurosignals 12:191-199.

Bresler T, Shapira M, Boeckers T, Dresbach T, Futter M, Garner CC, Rosenblum K, Gundelfinger ED, Ziv NE (2004) Postsynaptic density assembly is fundamentally different from presynaptic active zone assembly. J Neurosci 24:1507-1520.

Carroll RC, Zukin RS (2002) NMDA-receptor trafficking and targeting: im- 
plications for synaptic transmission and plasticity. Trends Neurosci 25:571-577.

Chen M, Hou X, Zhang G (2003) Tyrosine kinase and tyrosine phosphatase participate in regulation of interactions of NMDA receptor subunit $2 \mathrm{~A}$ with Src and Fyn mediated by PSD-95 after transient brain ischemia. Neurosci Lett 339:29-32.

Chen Y, Beffert U, Ertunc M, Tang TS, Kavalali ET, Bezprozvanny I, Herz J (2005) Reelin modulates NMDA receptor activity in cortical neurons. J Neurosci 25:8209-8216.

Chergui K, Svenningsson P, Greengard P (2004) Cyclin-dependent kinase 5 regulates dopaminergic and glutamatergic transmission in the striatum. Proc Natl Acad Sci USA 101:2191-2196.

Cheung HH, Gurd JW (2001) Tyrosine phosphorylation of the N-methylD-aspartate receptor by exogenous and postsynaptic density-associated Src-family kinases. J Neurochem 78:524-534.

Cheung ZH, Fu AK, Ip NY (2006) Synaptic roles of Cdk5: implications in higher cognitive functions and neurodegenerative diseases. Neuron 50:13-18.

Cruz JC, Tsai LH (2004) Cdk5 deregulation in the pathogenesis of Alzheimer's disease. Trends Mol Med 10:452-458.

Cull-Candy S, Brickley S, Farrant M (2001) NMDA receptor subunits: diversity, development and disease. Curr Opin Neurobiol 11:327-335.

Dhavan R, Tsai LH (2001) A decade of CDK5. Nat Rev Mol Cell Biol 2:749-759.

Dingledine R, Borges K, Bowie D, Traynelis SF (1999) The glutamate receptor ion channels. Pharmacol Rev 51:7-61.

Dunah AW, Standaert DG (2001) Dopamine D1 receptor-dependent trafficking of striatal NMDA glutamate receptors to the postsynaptic membrane. J Neurosci 21:5546-5558.

Fischer A, Sananbenesi F, Pang PT, Lu B, Tsai LH (2005) Opposing roles of transient and prolonged expression of p25 in synaptic plasticity and hippocampus-dependent memory. Neuron 48:825-838.

Fong DK, Rao A, Crump FT, Craig AM (2002) Rapid synaptic remodeling by protein kinase $\mathrm{C}$ : reciprocal translocation of NMDA receptors and calcium/calmodulin-dependent kinase II. J Neurosci 22:2153-2164.

Friedman HV, Bresler T, Garner CC, Ziv NE (2000) Assembly of new individual excitatory synapses: time course and temporal order of synaptic molecule recruitment. Neuron 27:57-69.

Fu WY, Chen Y, Sahin M, Zhao XS, Shi L, Bikoff JB, Lai KO, Yung WH, Fu AK, Greenberg ME, Ip NY (2007) Cdk5 regulates EphA4-mediated dendritic spine retraction through an ephexin1-dependent mechanism. Nat Neurosci 10:67-76.

Gao CY, Stepp MA, Fariss R, Zelenka P (2004) Cdk5 regulates activation and localization of Src during corneal epithelial wound closure. J Cell Sci 117:4089-4098.

Grant SG, O’Dell TJ, Karl KA, Stein PL, Soriano P, Kandel ER (1992) Impaired long-term potentiation, spatial learning, and hippocampal development in fyn mutant mice. Science 258:1903-1910.

Hall RA, Soderling TR (1997) Differential surface expression and phosphorylation of the $N$-methyl-D-aspartate receptor subunits NR1 and NR2 in cultured hippocampal neurons. J Biol Chem 272:4135-4140.

Hallett PJ, Spoelgen R, Hyman BT, Standaert DG, Dunah AW (2006) Dopamine D1 activation potentiates striatal NMDA receptors by tyrosine phosphorylation-dependent subunit trafficking. J Neurosci 26:4690-4700

Hardingham GE, Bading H (2003) The Yin and Yang of NMDA receptor signalling. Trends Neurosci 26:81-89.

Hawasli AH, Benavides DR, Nguyen C, Kansy JW, Hayashi K, Chambon P, Greengard P, Powell CM, Cooper DC, Bibb JA (2007) Cyclindependent kinase 5 governs learning and synaptic plasticity via control of NMDAR degradation. Nat Neurosci 10:880-886.

Hou XY, Zhang GY, Zong YY (2003) Suppression of postsynaptic density protein 95 expression attenuates increased tyrosine phosphorylation of NR2A subunits of $N$-methyl-D-aspartate receptors and interactions of Src and Fyn with NR2A after transient brain ischemia in rat hippocampus. Neurosci Lett 343:125-128.

Huang Y, Lu W, Ali DW, Pelkey KA, Pitcher GM, Lu YM, Aoto H, Roder JC, Sasaki T, Salter MW, MacDonald JF (2001) CAKbeta/Pyk2 kinase is a signaling link for induction of long-term potentiation in CA1 hippocampus. Neuron 29:485-496.

Hunter T (1987) A tail of two src's: mutatis mutandis. Cell 49:1-4.
Kalia LV, Salter MW (2003) Interactions between Src family protein tyrosine kinases and PSD-95. Neuropharmacology 45:720-728.

Kalia LV, Pitcher GM, Pelkey KA, Salter MW (2006) PSD-95 is a negative regulator of the tyrosine kinase Src in the NMDA receptor complex. EMBO J 25:4971-4982.

Kerokoski P, Suuronen T, Salminen A, Soininen H, Pirttila T (2004) Both $N$-methyl-D-aspartate (NMDA) and non-NMDA receptors mediate glutamate-induced cleavage of the cyclin-dependent kinase $5(\mathrm{cdk} 5)$ activator p35 in cultured rat hippocampal neurons. Neurosci Lett 368:181-185.

Kohr G, Seeburg PH (1996) Subtype-specific regulation of recombinant NMDA receptor-channels by protein tyrosine kinases of the Src family. J Physiol (Lond) 492:445-452.

Kornau HC, Schenker LT, Kennedy MB, Seeburg PH (1995) Domain interaction between NMDA receptor subunits and the postsynaptic density protein PSD-95. Science 269:1737-1740.

Kumari M, Ticku MK (2000) Regulation of NMDA receptors by ethanol. Prog Drug Res 54:152-189.

Lau CG, Zukin RS (2007) NMDA receptor trafficking in synaptic plasticity and neuropsychiatric disorders. Nat Rev Neurosci 8:413-426.

Lavezzari G, McCallum J, Lee R, Roche KW (2003) Differential binding of the AP-2 adaptor complex and PSD-95 to the C-terminus of the NMDA receptor subunit NR2B regulates surface expression. Neuropharmacology 4:729-737.

Lavezzari G, McCallum J, Dewey CM, Roche KW (2004) Subunit-specific regulation of NMDA receptor endocytosis. J Neurosci 24:6383-6391.

Lee SY, Wenk MR, Kim Y, Nairn AC, De Camilli P (2004) Regulation of synaptojanin 1 by cyclin-dependent kinase 5 at synapses. Proc Natl Acad Sci USA 101:546-551.

Lee SY, Voronov S, Letinic K, Nairn AC, Di Paolo G, De Camilli P (2005) Regulation of the interaction between PIPKI gamma and talin by prolinedirected protein kinases. J Cell Biol 168:789-799.

Li B, Chen N, Luo T, Otsu Y, Murphy TH, Raymond LA (2002) Differential regulation of synaptic and extra-synaptic NMDA receptors. Nat Neurosci 5:833-834.

Li B, Otsu Y, Murphy TH, Raymond LA (2003) Developmental decrease in NMDA receptor desensitization associated with shift to synapse and interaction with postsynaptic density-95. J Neurosci 23:11244-11254.

Li BS, Sun MK, Zhang L, Takahashi S, Ma W, Vinade L, Kulkarni AB, Brady RO, Pant HC (2001) Regulation of NMDA receptors by cyclindependent kinase-5. Proc Natl Acad Sci USA 98:12742-12747.

Lim IA, Hall DD, Hell JW (2002) Selectivity and promiscuity of the first and second PDZ domains of PSD-95 and synapse-associated protein 102. J Biol Chem 277:21697-21711.

Lin B, Arai AC, Lynch G, Gall CM (2003) Integrins regulate NMDA receptor-mediated synaptic currents. J Neurophysiology 89:2874-2878.

Lu WY, Xiong ZG, Lei S, Orser BA, Dudek E, Browning MD, MacDonald JF (1999) G-protein-coupled receptors act via protein kinase C and Src to regulate NMDA receptors. Nat Neurosci 2:331-338.

Lu YM, Roder JC, Davidow J, Salter MW (1998) Src activation in the induction of long-term potentiation in CA1 hippocampal neurons. Science 279:1363-1367.

Montgomery JM, Madison DV (2002) State-dependent heterogeneity in synaptic depression between pyramidal cell pairs, Neuron 33:765-777.

Morabito MA, Sheng M, Tsai LH (2004) Cyclin-dependent kinase 5 phosphorylates the $N$-terminal domain of the postsynaptic density protein PSD-95 in neurons. J Neurosci 24:865-876.

Nakazawa T, Komai S, Tezuka T, Hisatsune C, Umemori H, Semba K, Mishina M, Manabe T, Yamamoto T (2001) Characterization of Fynmediated tyrosine phosphorylation sites on GluR epsilon 2 (NR2B) subunit of the N-methyl-D-aspartate receptor. J Biol Chem 276:693-699.

Nakazawa T, Komai S, Watabe AM, Kiyama Y, Fukaya M, Arima-Yoshida F, Horai R, Sudo K, Ebine K, Delawary M, Goto J, Umemori H, Tezuka T, Iwakura Y, Watanabe M, Yamamoto T, Manabe T (2006) NR2B tyrosine phosphorylation modulates fear learning as well as amygdaloid synaptic plasticity. EMBO J 25:2867-2877.

Niethammer M, Kim E, Sheng M (1996) Interaction between the C terminus of NMDA receptor subunits and multiple members of the PSD-95 family of membrane-associated guanylate kinases. J Neurosci 16:2157-2163.

Nong Y, Huang YQ, Ju W, Kalia LV, Ahmadian G, Wang YT, Salter MW 
(2003) Glycine binding primes NMDA receptor internalization. Nature 422:302-307.

Nong Y, Huang YQ, Salter MW (2004) NMDA receptors are movin' in, Curr Opin Neurobiol 14:353-361.

Ohshima T, Ogura H, Tomizawa K, Hayashi K, Suzuki H, Saito T, Kamei H, Nishi A, Bibb JA, Hisanaga S, Matsui H, Mikoshiba K (2005) Impairment of hippocampal long-term depression and defective spatial learning and memory in p35 mice. J Neurochem 94:917-925.

Patel J, Mooslehner KA, Chan PM, Emson PC, Stamford JA (2003) Presynaptic control of striatal dopamine neurotransmission in adult vesicular monoamine transporter 2 (VMAT2) mutant mice. J Neurochem 85:898-910.

Perez-Otano I, Ehlers MD (2005) Homeostatic plasticity and NMDA receptor trafficking. Trends Neurosci 28:229-238.

Perez-Otano I, Lujan R, Tavalin SJ, Plomann M, Modregger J, Liu XB, Jones EG, Heinemann SF, Lo DC, Ehlers MD (2006) Endocytosis and synaptic removal of NR3A-containing NMDA receptors by PACSIN1/syndapin 1 . Nat Neurosci 9:611-621.

Prybylowski K, Chang K, Sans N, Kan L, Vicini S, Wenthold RJ (2005) The synaptic localization of NR2B-containing NMDA receptors is controlled by interactions with PDZ proteins and AP-2. Neuron 47:845-857.

Roche KW, Standley S, McCallum J, Dune Ly C, Ehlers MD, Wenthold RJ (2001) Molecular determinants of NMDA receptor internalization. Nat Neurosci 4:794-802.

Rosenblum K, Dudai Y, Richter-Levin G (1996) Long-term potentiation increases tyrosine phosphorylation of the $N$-methyl-D-aspartate receptor subunit $2 \mathrm{~B}$ in rat dentate gyrus in vivo. Proc Natl Acad Sci USA 93:10457-10460.

Ross CA, Margolis RL, Reading SA, Pletnikov M, Coyle JT (2006) Neurobiology of schizophrenia. Neuron 52:139-153.

Rostas JA, Brent VA, Voss K, Errington ML, Bliss TV, Gurd JW (1996) Enhanced tyrosine phosphorylation of the $2 \mathrm{~B}$ subunit of the $N$-methyl-Daspartate receptor in long-term potentiation. Proc Natl Acad Sci USA 93:10452-10456.

Salter MW, Kalia LV (2004) Src kinases: a hub for NMDA receptor regulation. Nat Rev Neurosci 5:317-328.

Schuman EM, Murase S (2003) Cadherins and synaptic plasticity: activitydependent cyclin-dependent kinase 5 regulation of synaptic beta-catenincadherin interactions. Philos Trans R Soc Lond B Biol Sci 358:749-756.

Scott DB, Michailidis I, Mu Y, Logothetis D, Ehlers MD (2004) Endocytosis and degradative sorting of NMDA receptors by conserved membraneproximal signals. J Neurosci 24:7096-7109.

Selkoe DJ (2002) Alzheimer's disease is a synaptic failure. Science 298:789-791.

Snyder EM, Philpot BD, Huber KM, Dong X, Fallon JR, Bear MF (2001) Internalization of ionotropic glutamate receptors in response to $\mathrm{mGluR}$ activation. Nat Neurosci 4:1079-1085.

Snyder EM, Nong Y, Almeida CG, Paul S, Moran T, Choi EY, Nairn AC, Salter
MW, Lombroso PJ, Gouras GK, Greengard P (2005) Regulation of NMDA receptor trafficking by amyloid-beta. Nat Neurosci 8:1051-1058.

Takasu MA, Dalva MB, Zigmond RE, Greenberg ME (2002) Modulation of NMDA receptor-dependent calcium influx and gene expression through EphB receptors. Science 295:491-495.

Tan TC, Valova VA, Malladi CS, Graham ME, Berven LA, Jupp OJ, Hansra G, McClure SJ, Sarcevic B, Boadle RA, Larsen MR, Cousin MA, Robinson PJ (2003) Cdk5 is essential for synaptic vesicle endocytosis. Nat Cell Biol 5:701-710.

Taylor JR, Lynch WJ, Sanchez H, Olausson P, Nestler EJ, Bibb JA (2007) Inhibition of Cdk5 in the nucleus accumbens enhances the locomotoractivating and incentive-motivational effects of cocaine. Proc Natl Acad Sci USA 104:4147-4152.

Tezuka T, Umemori H, Akiyama T, Nakanishi S, Yamamoto T (1999) PSD-95 promotes Fyn-mediated tyrosine phosphorylation of the $N$-methyl-D-aspartate receptor subunit NR2A. Proc Natl Acad Sci USA 96:435-440.

Tomizawa K, Sunada S, Lu YF, Oda Y, Kinuta M, Ohshima T, Saito T, Wei FY, Matsushita M, Li ST, Tsutsui K, Hisanaga S, Mikoshiba K, Takei K, Matsui H (2003) Cophosphorylation of amphiphysin I and dynamin I by Cdk5 regulates clathrin-mediated endocytosis of synaptic vesicles. J Cell Biol 163:813-824.

Vissel B, Krupp JJ, Heinemann SF, Westbrook GL (2001) A use-dependent tyrosine dephosphorylation of NMDA receptors is independent of ion flux. Nat Neurosci 4:587-596.

Wang Q, Walsh DM, Rowan MJ, Selkoe DJ, Anwyl R (2004) Block of longterm potentiation by naturally secreted and synthetic amyloid betapeptide in hippocampal slices is mediated via activation of the kinases c-Jun $N$-terminal kinase, cyclin-dependent kinase 5, and p38 mitogenactivated protein kinase as well as metabotropic glutamate receptor type 5. J Neurosci 24:3370-3378.

Wang YT, Salter MW (1994) Regulation of NMDA receptors by tyrosine kinases and phosphatases. Nature 369:233-235.

Washbourne P, Bennett JE, McAllister AK (2002) Rapid recruitment of NMDA receptor transport packets to nascent synapses. Nat Neurosci 5:751-759.

Wei FY, Tomizawa K, Ohshima T, Asada A, Saito T, Nguyen C, Bibb JA, Ishiguro K, Kulkarni AB, Pant HC, Mikoshiba K, Matsui H, Hisanaga S (2005) Control of cyclin-dependent kinase 5 (Cdk5) activity by glutamatergic regulation of p35 stability. J Neurochem 93:502-512.

Wenthold RJ, Prybylowski K, Standley S, Sans N, Petralia RS (2003) Trafficking of NMDA receptors. Annu Rev Pharmacol Toxicol 43:335-358.

Yu XM, Askalan R, Keil GJ, Salter MW (1997) NMDA channel regulation by channel-associated protein tyrosine kinase Src. Science 275:674-678.

Zhang X, Lee TH, Davidson C, Lazarus C, Wetsel WC, Ellinwood EH (2007) Reversal of cocaine-induced behavioral sensitization and associated phosphorylation of the NR2B and GluR1 subunits of the NMDA and AMPA receptors. Neuropsychopharmacology 32:377-387. 